\title{
Molecular Detection of Peronospora variabilis in Quinoa Seed and Phylogeny of the Quinoa Downy Mildew Pathogen in South America and the United States
}

\author{
Anna L. Testen, María del Mar Jiménez-Gasco, José B. Ochoa, and Paul A. Backman
}

First, second, and fourth authors: Department of Plant Pathology and Environmental Microbiology, The Pennsylvania State University, University Park; and third author: INIAP Santa Catalina Station, Quito, Ecuador. Current address of A. L. Testen: Department of Plant Pathology, The Ohio State University OARDC, Wooster. Accepted for publication 30 October 2013.

\begin{abstract}
Testen, A. L., Jiménez-Gasco, M. M., Ochoa, J. B., and Backman, P. A. 2014. Molecular detection of Peronospora variabilis in quinoa seed and phylogeny of the quinoa downy mildew pathogen in South America and the United States. Phytopathology 104:379-386.

Quinoa (Chenopodium quinoa) is an important export of the Andean region, and its key disease is quinoa downy mildew, caused by Peronospora variabilis. P. variabilis oospores can be seedborne and rapid methods to detect seedborne $P$. variabilis have not been developed. In this research, a polymerase chain reaction (PCR)-based detection method was developed to detect seedborne $P$. variabilis and a sequencing-based method was used to validate the PCR-based method. $P$. variabilis was detected in 31 of 33 quinoa seed lots using the PCR-based method and in 32 of 33 quinoa seed lots using the sequencing-based method. Thirty-one of the quinoa seed lots tested in this study were sold for human con-

sumption, with seed originating from six different countries. Internal transcribed spacer (ITS) and cytochrome $c$ oxidase subunit 2 (COX2) phylogenies were examined to determine whether geographical differences occurred in P. variabilis populations originating from Ecuador, Bolivia, and the United States. No geographical differences were observed in the ITS-derived phylogeny but the COX2 phylogeny indicated that geographical differences existed between U.S. and South American samples. Both ITS and COX2 phylogenies supported the existence of a Peronospora sp., distinct from $P$. variabilis, that causes systemic-like downy mildew symptoms on quinoa in Ecuador. The results of these studies allow for a better understanding of $P$. variabilis populations in South America and identified a new causal agent for quinoa downy mildew. The PCR-based seed detection method allows for the development of $P$. variabilis-free quinoa seed, which may prove important for management of quinoa downy mildew.
\end{abstract}

Quinoa (Chenopodium quinoa Willd.) is the most recent Andean crop to garner global attention as a food crop $(4,8,17,25)$. The majority of quinoa production occurs in the Andean countries of Bolivia, Peru, and Ecuador (35), but production has spread from South America to Europe, North America, and beyond (8). In major quinoa-producing regions, the crop's key disease is quinoa downy mildew (10), caused by Peronospora variabilis Gäum (formerly $P$. farinosa f. sp. chenopodii Byford) (5), a heterothallic (9) oomycete. Quinoa downy mildew epidemics greatly reduce yield (10), and under experimental conditions, $P$. variabilis reduced seed yields by at least $33 \%$ in resistant varieties and up to $99 \%$ in susceptible varieties (11). Although quinoa downy mildew is such an important disease of quinoa, there are still several key research areas that are underdeveloped, including molecular detection of $P$. variabilis and the phylogenetic structure of the causal agent of quinoa downy mildew.

Quinoa downy mildew has been reported in countries outside of quinoa's native range, including Canada (34), Denmark (12), India (28), and, most recently, the United States (Pennsylvania in 2011) (33). The original source of inoculum for each $P$. variabilis introduction is unknown, but the pathogen was likely introduced on infected seed. The oospores of $P$. variabilis can be seedborne and remain viable (1), and current oospore detection methods rely on germinating seed under disease conducive conditions (14) or examining seed microscopically (14). If oospores are detectable

Corresponding author: A. L. Testen; E-mail address: testen.2@ osu.edu

http://dx.doi.org/10.1094/PHYTO-07-13-0198-R

(c) 2014 The American Phytopathological Society by these two methods, molecular detection of $P$. variabilis in quinoa seed should also be possible. Molecular methods have been developed to detect Peronospora spp. in other seed, including basil (3) and poppy (29). Molecular methods allow for screening more seed for the presence of $P$. variabilis in less time and allow for the standardization of methods to detect the pathogen in seed. These techniques could be further standardized to create certified $P$. variabilis-free quinoa seed.

The genetic diversity of $P$. variabilis has not been properly addressed, but some studies have suggested geographic differences in pathogen populations between European and South American populations based on phylogenies of the internal transcribed spacer (ITS) region of the ribosomal DNA (rDNA) (5) and universally primed polymerase chain reaction (PCR) (13). Both studies indicated that European (Danish) samples and South American (Ecuadorean, Peruvian, and Bolivian) samples formed separate clusters within the same species $(5,13)$. Samples from South America, especially Bolivia, were underrepresented in these studies, and it is possible that the true diversity of the quinoa downy mildew pathogen in South America was not fully explored in previous studies. Also, downy mildew samples from closely related Chenopodium weeds have not been examined, likely because previous studies have indicated a limited host range for the quinoa downy mildew pathogen $(1,10)$. No previous studies have used a multigene phylogeny to examine the same population of $P$. variabilis from quinoa; however, multigene analyses have been used extensively to determine the phylogenies of Peronospora spp. (20) and other oomycetes $(2,27)$. Phylogenetic studies of Peronospora spp. have been conducted using data obtained from ITS (19), large subunit rDNA (21), cyto- 
chrome $c$ oxidase subunit 2 (COX2) $(22,23)$, NADH dehydrogenase subunit 1 (20), and $\beta$-tubulin (20). Due to the obligate nature of $P$. variabilis, it is important to select genetic markers with primers that will only amplify $P$. variabilis DNA, even in the presence of DNA from plant material or other fungi.

The objectives of this research were twofold: to (i) develop a method to rapidly screen quinoa seed for $P$. variabilis and (ii) better understand the phylogenetic relationship of isolates of the quinoa downy mildew pathogen from disease samples collected from endemic and introduced regions.

In this research, we designed specific primers for $P$. variabilis in order to develop a rapid detection method for $P$. variabilis in quinoa seed, including quinoa seed imported for human consumption. This PCR-based method targeted the ITS region and was supported by a sequencing-based method of seed detection of the quinoa downy mildew pathogen. We also examined the phylogenies of two regions, ITS (6) and COX2 (23), from quinoa downy mildew samples collected in Bolivia, Ecuador, and the United States to determine whether genetic differences in $P$. variabilis existed based on geography.

\section{MATERIALS AND METHODS}

Seed, plant, and $\boldsymbol{P}$. variabilis samples. For seed screening studies, 33 seed lots of domestic and imported quinoa seed, most

TABLE 1. Detection of Peronospora variabilis in quinoa seed using sequencing and polymerase chain reaction (PCR)-based methods developed in this study

\begin{tabular}{|c|c|c|c|}
\hline Seed source & $\begin{array}{l}\text { Country of } \\
\text { origin }\end{array}$ & $\begin{array}{c}\text { Sequencing } \\
\text { confirmation }^{\mathrm{a}, \mathrm{b}}\end{array}$ & $\begin{array}{c}\text { PCR } \\
\text { amplification }^{\mathrm{c}}\end{array}$ \\
\hline AEP & Bolivia & $+/+$ & $-1+$ \\
\hline AER & Bolivia & $+/+$ & $+/+$ \\
\hline AEM & Bolivia & $+/+$ & $+/+$ \\
\hline WegW & Bolivia & $+/+$ & $+/+$ \\
\hline WBT & Bolivia & $+/+$ & $+/+$ \\
\hline WBR & Bolivia & $-1+$ & $-1+$ \\
\hline WegR & Bolivia & $+/+$ & $+/+$ \\
\hline TJW & Bolivia & $+/+$ & $+/+$ \\
\hline TJR & Bolivia & $+/+$ & $+/+$ \\
\hline TJT & Bolivia & $+/+$ & $+/+$ \\
\hline TRU & Bolivia & $+/+$ & $+/+$ \\
\hline WegB & Bolivia & $+/+$ & $+/+$ \\
\hline EOR & Bolivia & $+/+$ & $+/+$ \\
\hline EOW & Ecuador & $+/+$ & $+/+$ \\
\hline AHMW & Bolivia & +/Muc & $+/+$ \\
\hline ICN & Bolivia & $+/+$ & $+/+$ \\
\hline $\mathrm{COC}$ & Bolivia & $+/+$ & $+/+$ \\
\hline BLO & Bolivia & $+/+$ & $+/+$ \\
\hline $\mathrm{ECW}$ & Bolivia & $+/+$ & $+/+$ \\
\hline AHW & Bolivia & $+/+$ & $+/+$ \\
\hline GIA & Bolivia & $+/+$ & $+/+$ \\
\hline NPB & Bolivia & $+/+$ & $+/+$ \\
\hline \multicolumn{4}{|l|}{ Chenopodium } \\
\hline pallidicaule (KAN) & Bolivia & $-1-$ & $-1+$ \\
\hline SAH & Peru & $+/$ Asp & $+/+$ \\
\hline PHW & Peru & $+/+$ & $+/+$ \\
\hline WGS & India & $+/+$ & $+/+$ \\
\hline SIS10 & United States & $-/$ Asp & $-1-$ \\
\hline SIS11 & United States & $+/+$ & $+/+$ \\
\hline WMW & United States & $+/+$ & $+/+$ \\
\hline WMB & United States & $-1+$ & $+/+$ \\
\hline C. album (CAL) & United States & $-1-$ & $-1-$ \\
\hline NQG & Canada & $+/+$ & $+1-$ \\
\hline NQB & Canada & $-1+$ & $+/+$ \\
\hline NQGO & Canada & $+/+$ & $-1-$ \\
\hline NQBO & Canada & $+/+$ & $+/+$ \\
\hline
\end{tabular}

${ }^{a}$ Results are shown for two replicates of each detection method; + indicates $P$. variabilis was detected, - indicates $P$. variabilis was not detected, Asp = sequencing reactions that amplified Aspergillus sp., and Muc = sequencing reactions that amplified Mucor sp.

b Sequencing confirmation of $P$. variabilis.

c PCR amplification of $P$. variabilis with primer set PV6. sold for human consumption, along with one seed lot each of C. pallidicaule (the Andean crop kañiwa) and C. album (lambsquarters), were tested in this study and each seed lot was tested twice. The quinoa seed analyzed in this study originated from six different countries: Bolivia, Ecuador, Peru, India, Canada, and the United States (Table 1).

For phylogenetic analysis, quinoa and Chenopodium spp. (C. album and Chenopodium sp.) leaf samples were collected from mature plants affected by downy mildew (Fig. 1, panels E and F) originating from Bolivia, Ecuador, and the United States (Table 2) during the 2011 to 2013 growing seasons. Samples were dried overnight at $55^{\circ} \mathrm{C}$ and stored at room temperature prior to DNA extraction. A single lesion was chosen from each leaf for DNA extraction. Sporangia samples collected in Ecuador in 2013 were preserved in $70 \%$ ethanol prior to DNA extraction. For Bolivian and U.S. samples, five extractions (leaves taken from different plants) were performed for each field. Total DNA was extracted using the Qiagen DNeasy Plant Mini Kit (Qiagen, Venlo, The Netherlands), following manufacturer's instructions.

In 2011, quinoa downy mildew was detected in Centre County and Lancaster County, PA (33). Samples were collected from both field research sites, and $P$. variabilis was maintained on detached quinoa 'Faro' (Siskiyou Seeds, Williams, OR) leaves (14) on water agar. Two lines of $P$. variabilis were maintained: the Rock Springs line (RS) (Centre County) and the Landisville line (LV) (Lancaster County). Mating tests were performed as described by Danielsen et al. (9).

Development of $\boldsymbol{P}$. variabilis-specific primers. $P$. variabilis ITS sequences were obtained from GenBank and from sequences obtained from RS and LV $P$. variabilis lines, following amplification using primer pair DC6/ITS4 (7) (Table 3). Primer set PV6 ( $P$. variabilis 6) (Table 3) was developed from the ITS consensus sequence using National Center for Biotechnology Information Primer Blast (37). Optimal PCR conditions for primer set PV6 consisted of the following: initial denaturation step of $94^{\circ} \mathrm{C}$ for $2 \mathrm{~min}$; followed by 32 cycles of a touchdown PCR (26) with denaturation at $95^{\circ} \mathrm{C}$ for $30 \mathrm{~s}$, annealing from 66 to $56^{\circ} \mathrm{C}$ for $45 \mathrm{~s}$, and elongation at $72^{\circ} \mathrm{C}$ for $90 \mathrm{~s}$; with a final elongation step of $72^{\circ} \mathrm{C}$ for $5 \mathrm{~min}$. PCR was carried out in $20-\mu \mathrm{l}$ reactions consisting of GoTaq green master mix (Promega Corporation, Madison, WI), $0.25 \mu \mathrm{M}$ each primer, and $1 \mu \mathrm{l}$ of template DNA. All reactions were carried out in an ExpressGene Gradient Cycler (Denville Scientific, Metuchen, NJ).

To assess primer specificity, primer set PV6 was tested to determine whether the primers would amplify DNA from P. farinosa f. sp. spinaciae on spinach, Bremia lactucae on lettuce, Pythium aphanidermatum (pure culture), Phytophthora infestans on tomato, Plasmopara obducens on impatiens, Peronospora tabacina on tobacco, P. manshurica on soybean, Plasmopara viticola on grape, or Pseudoperonospora cubensis on cucumber.

To determine primer sensitivity, template DNA from sporangia was amplified using the DC6/ITS4 primer pair. PCR products were run on agarose gels and stained with ethidium bromide, and bands corresponding to Peronospora variabilis DNA were excised from the gel and purified using the E.Z.N.A gel extraction kit (Omega Bio-Tek, Norcross, GA) to ensure no carryover of plant DNA. Purified $P$. variabilis DNA was quantified using a NanoDrop2000 (Thermo Fisher Scientific, Waltham, MA) and serial DNA dilutions were amplified using primer pair PV6 to determine primer sensitivity.

Detection of $\boldsymbol{P}$. variabilis in quinoa seed. Seed wash methods for microscopically examining quinoa seed for oospores $(14,24)$ were adapted to screen seed molecularly. Briefly, $5 \mathrm{~g}(\approx 1,300$ to 1,500 seeds) of quinoa seed was stirred in sterile milliQ water for $30 \mathrm{~min}$ at maximum speed. The seed wash suspension was filtered through sterile cheesecloth and centrifuged for $5 \mathrm{~min}$ at 4,000 rpm. The obtained pellet was transferred to a tube of Lysing Matrix A 
(MP Biomedicals, Solon, $\mathrm{OH}$ ) and lysed in a FastPrep-24 Instrument (MP Biomedicals) for two cycles of $45 \mathrm{~s}$ at speed 4.5. DNA was then extracted using a Qiagen DNeasy Plant Mini Kit. $P$. variabilis-specific primer set PV6 was then used to screen seed for $P$. variabilis. The optimal PCR conditions for this reaction are described above. Amplification was determined by running the PCR products on an agarose gel, followed by ethidium bromide staining.

To validate the specific primer PCR-based method, a sequencing-based detection method was also used to screen quinoa seed. A semi-nested PCR (7) was used to obtain DNA from the extracted seed washes for DNA sequencing. This semi-nested PCR consisted of 25- $\mu$ l reactions of GoTaq green master mix, $0.2 \mu \mathrm{M}$ each primer (first reaction: ITS4/DC6 and second reac- tion: ITS4/ITS6), and $5 \mu \mathrm{l}$ of each template. PCR reactions were performed according to Cooke et al. (7). PCR products were cleaned with ExoSap IT (Affymetrix/USB, Santa Clara, CA) and submitted for sequencing at the Penn State Genomics Core Facility. Products were sequenced unidirectionally with primer DC6 using an Applied Biosystems 3730XL DNA Analyzer (Applied Biosystems, Foster City, CA).

To validate both molecular detection methods, some seed wash pellets were treated with $5 \mathrm{ml}$ of $1 \mathrm{M} \mathrm{KOH}$ for $16 \mathrm{~h}$ (14) to visually check for the presence of oospores using a light microscope.

Phylogenetics of the quinoa downy mildew pathogen. Two regions, ITS (7) and COX2 (22,23), were amplified for phylogenetic study of the causal agent of quinoa downy mildew. For both genes, 25- $\mu$ PCR reactions consisted of GoTaq green master

TABLE 2. Samples of quinoa downy mildew used in this study by host, country and region of origin, and year collected

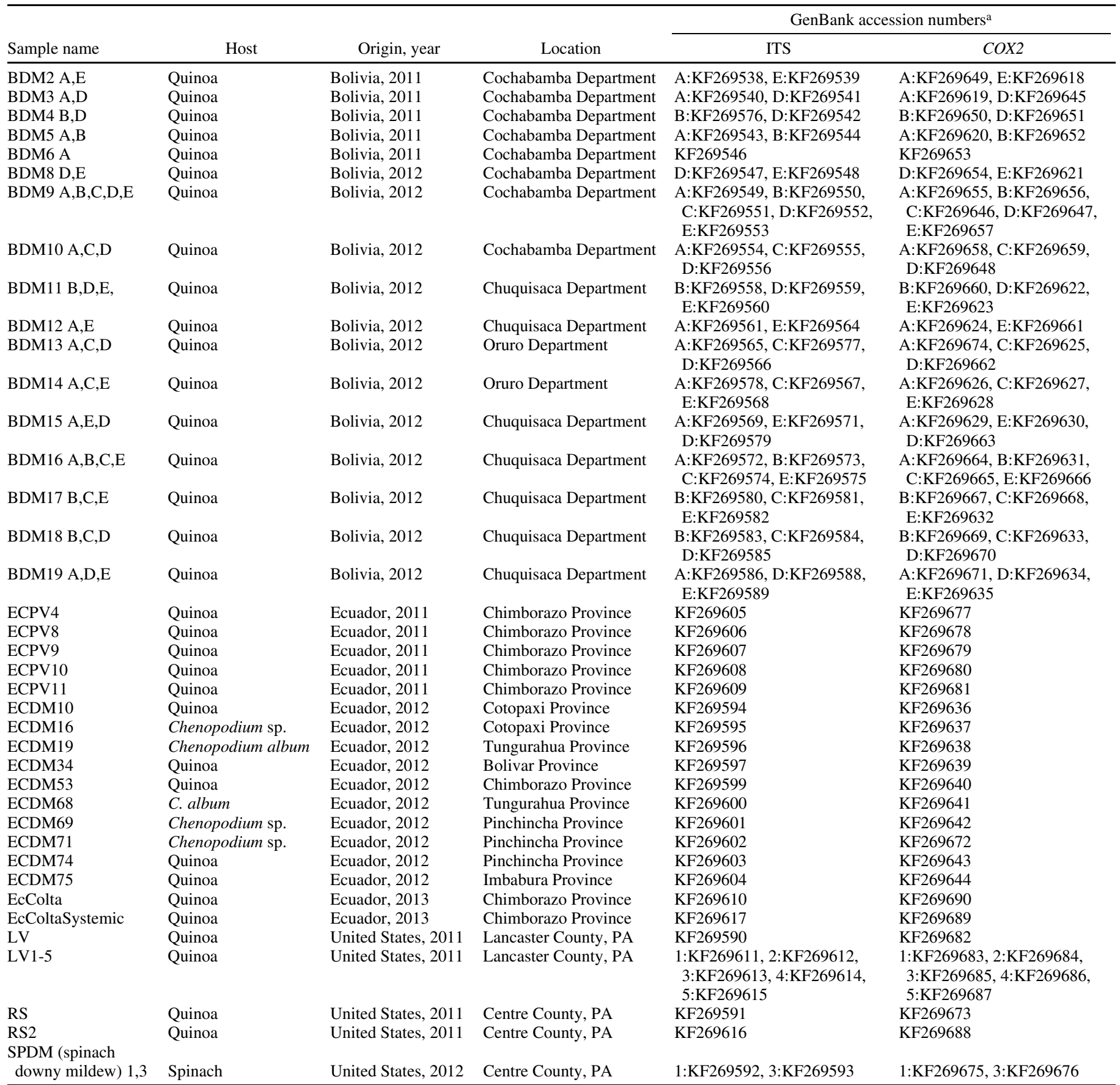

${ }^{a}$ GenBank accession numbers are given for multiple samples collected from different quinoa plants in a field. For Bolivian samples, up to five samples were collected (A to E) and, for U.S. samples, up to five were also collected (1 to 5). ITS = internal transcribed spacer and COX2 = cytochrome $c$ oxidase subunit 2. 
mix, $0.2 \mu \mathrm{M}$ each primer, and $5 \mu \mathrm{l}$ of template DNA. ITS sequences were amplified using primers ITS4/DC6, with PCR conditions described by Cooke et al. (7). Amplification of COX2 used the peronosporomycete forward/reverse primers (22), with PCR conditions of $96^{\circ} \mathrm{C}$ for $4 \mathrm{~min} ; 30$ cycles of $96^{\circ} \mathrm{C}$ for $30 \mathrm{~s}$, $50^{\circ} \mathrm{C}$ for $30 \mathrm{~s}$, and $72^{\circ} \mathrm{C}$ for $1 \mathrm{~min}$; followed by a final elongation step of $72^{\circ} \mathrm{C}$ for $4 \mathrm{~min}$. PCR products were visualized on agarose gels following staining with ethidium bromide. PCR products were cleaned with ExoSAP-IT prior to sequencing.

PCR products were sequenced bidirectionally using primers ITS4/DC6 or primers peronosporomycete forward/reverse. Obtained sequences were aligned using MUSCLE (16) in MEGA5.10 (32) and alignments were manually checked. Maximum likelihood (ML) trees were generated in MEGA5.10 and tested with 1,000 bootstrap replicates. An ML ITS tree was generated using the Hasegawa-Kishino-Yano model with invariant sites, while the ML COX2 tree was generated using the Tamura 3-parameter model with invariant sites. Pythium monospermum was used to root both trees (21).

\section{RESULTS}

Primer specificity and sensitivity. Primer set PV6 only amplified DNA from P. variabilis (Fig. 2) and consistently produced a 278-bp amplicon. The touchdown PCR protocol was required to avoid amplification of the closely related Peronospora farinosa $\mathrm{f}$. sp. spinaciae, because only a 3-nucleotide difference existed between both species in the annealing site of the reverse primer. The touchdown PCR also allowed for high primer sensitivity, with a detection limit for $P$. variabilis DNA of $10 \mathrm{fg} / \mu \mathrm{l}$ (Fig. 3). This primer sensitivity is for pure $P$. variabilis DNA and may be reduced in the presence of other DNA such as plant or fungal DNA.

Molecular detection of $P$. variabilis in quinoa seed. Thirtythree seed lots of quinoa were screened using the $P$. variabilis-

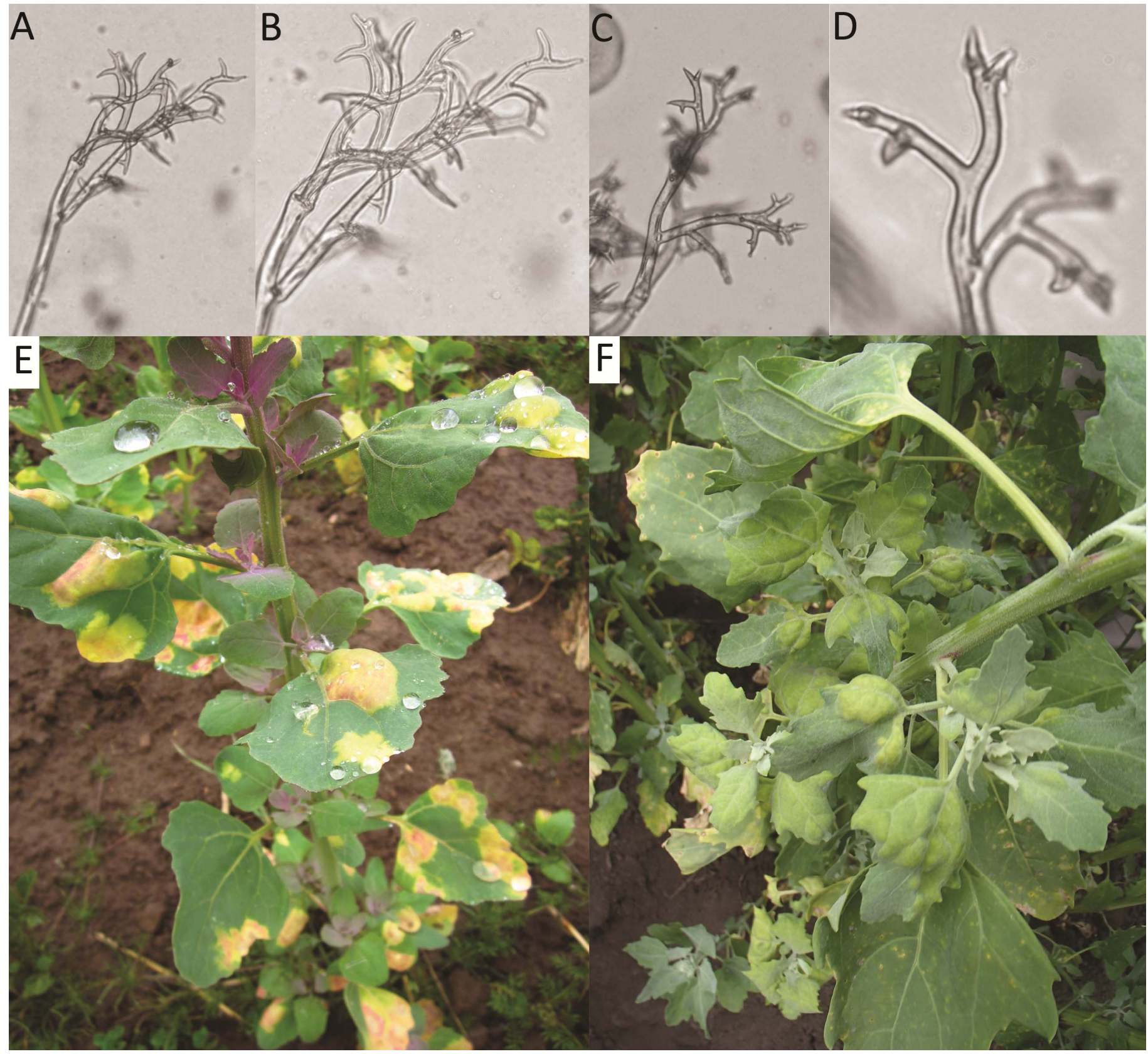

Fig. 1. A and B, Sporangiophore of Peronospora variabilis; C and D, sporangiophore of a putative Peronospora sp. which causes systemic-like downy mildew symptoms; E, typical symptoms of quinoa downy mildew, including yellow-pink foliar lesions with sporulation; $\mathbf{F}$, atypical symptoms of quinoa downy mildew, including stunting, foliar cupping, chlorosis, and extremely dense sporulation. 
specific primer PV6 designed in this study. P. variabilis was detected in 31 of 33 seed lots (Table 1; Fig. 4) in at least one of the two replicates. In 28 seed lots, $P$. variabilis was detected in both replicates (Table 1). $P$. variabilis was never detected in seed of $C$. album and was detected once in seed of $C$. pallidicaule (Table 1). Oospores were also visually observed in several seed lots, along with sporangia similar to those of $P$. variabilis (Fig. 5).

A sequencing-based approach was used to validate the PCRbased method and, in this approach, a semi-nested PCR was used (primers ITS4/DC6 for first reaction and ITS4/ITS6 for second reaction) to generate amplicons for sequencing. $P$. variabilis was detected in 32 of 33 samples of quinoa and was never detected in seed of $C$. album or C. pallidicaule; therefore, PCR-based detection of $P$. variabilis in $C$. pallidicaule seed was not validated (Table 1). The use of the semi-nested procedure to amplify the

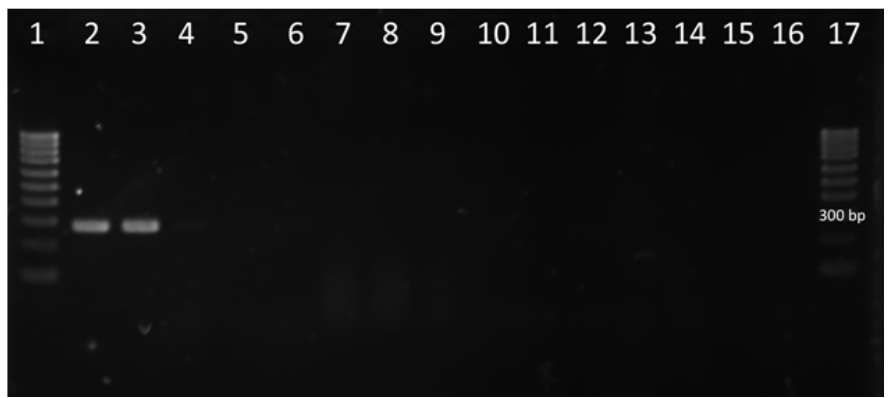

Fig. 2. Specificity of PV6 primers (repeated species are technical replicates). Lane 1, 1-kb ladder; lane 2, Peronospora variabilis on quinoa; lane 3, $P$. variabilis on quinoa; lane 4, $P$. farinosa $\mathrm{f}$. sp. spinaciae on spinach; lane $5, P$. farinosa f. sp. spinaciae on spinach; lane 6 , P. farinosa f. sp. spinaciae on spinach; lane 7, P. tabacina on tobacco; lane 8, P. manshurica on soybean; lane 9, Plasmopara viticola on grape; lane 10, P. obducens on impatiens; lane 11, Pseudoperonospora cubensis on cucumber; lane 12, Bremia lactucae on lettuce; lane 13, Phytophthora infestans on tomato; lane 14, Pythium aphanidermatum; lane 15, asymptomatic quinoa tissue; lane 16, negative control; lane 17, 1-kb ladder.

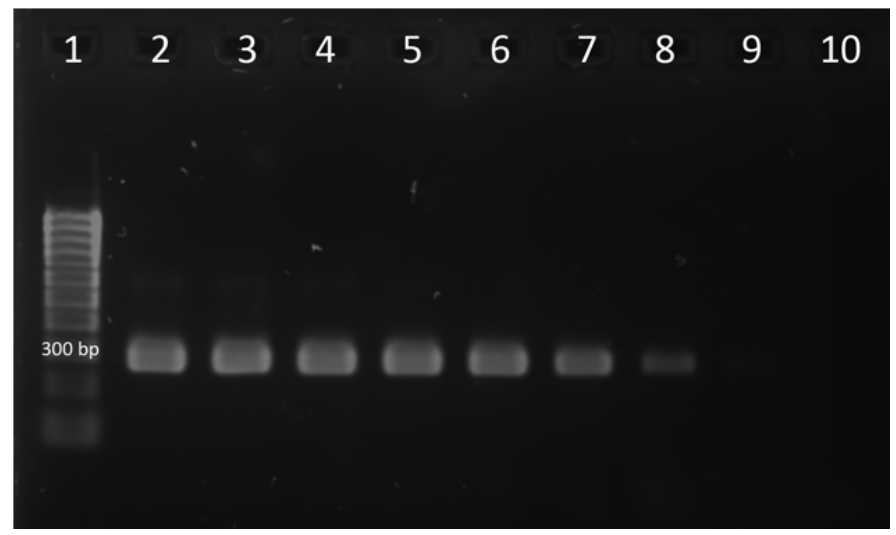

Fig. 3. Sensitivity of primer set PV6 for detection of Peronospora variabilis. Lane 2, $10 \mathrm{ng} / \mu \mathrm{l}$; lane 3, $1 \mathrm{ng} / \mu \mathrm{l}$; lane 4, $100 \mathrm{pg} / \mu \mathrm{l}$; lane 5, $10 \mathrm{pg} / \mu \mathrm{l}$; lane 6 , $1 \mathrm{pg} / \mu \mathrm{l}$; lane 7, $100 \mathrm{fg} / \mu \mathrm{l}$; lane $8,10 \mathrm{fg} / \mu \mathrm{l}$; lane $9,1 \mathrm{fg} / \mu \mathrm{l}$; lane $10,100 \mathrm{ag} / \mu \mathrm{l}$.
ITS region of Peronospora spp. (7) led to chimeric amplicons which had the $C$. quinoa ITS region as the first portion of the sequence, followed by the $P$. variabilis ITS region. This chimeric amplicon occurred in all seed lots tested. If $P$. variabilis was not detected by sequencing, only the $C$. quinoa ITS region was present in the sequenced amplicon.

SIS10, from the United States, was the only seed lot in which $P$. variabilis was never detected. However, the sequencing-based detection method amplified Aspergillus sp. DNA in this seed lot, and also in the SAH seed lot from Peru. However, $P$. variabilis was never detected with the PCR-based detection method in the SIS10 seed lot. SIS10 and SIS11 were the only seed lots sold for planting; all other seed lots were sold for human consumption.

Phylogeny of the quinoa downy mildew pathogen. Highquality sequences with no polymorphisms were obtained and used to construct phylogenetic trees based on ITS and COX2 sequences. The ITS phylogenetic tree (Fig. 6) showed little variation among quinoa downy mildew samples regardless of their South or North American origin. Only a single distinct clade (100\% bootstrap support) that included all $P$. variabilis from quinoa is shown in this tree. This clade also includes downy

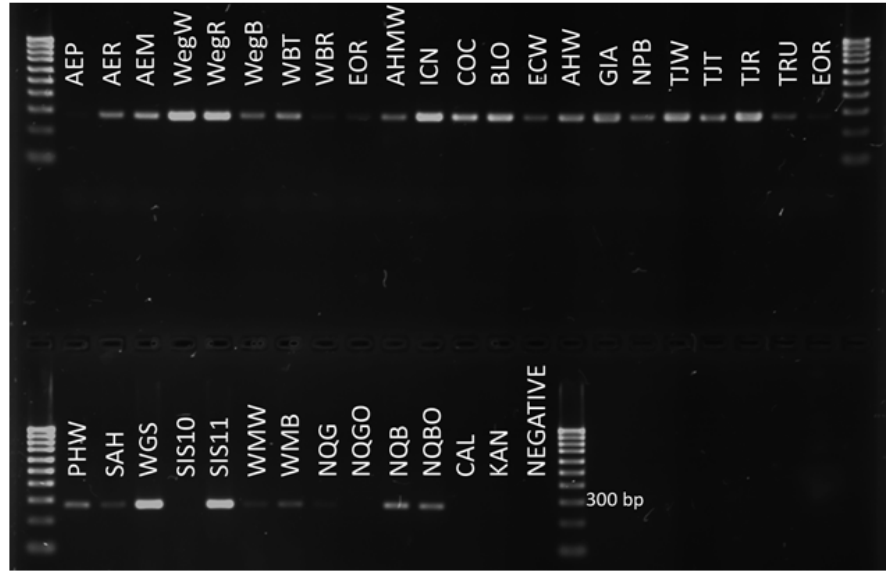

Fig. 4. Electrophoresis gel showing results of polymerase chain reaction-based detection of Peronospora variabilis in quinoa seed washes. Product size is approximately $278 \mathrm{bp}$. Codes for the sources of the seed washes are found in Table 1.

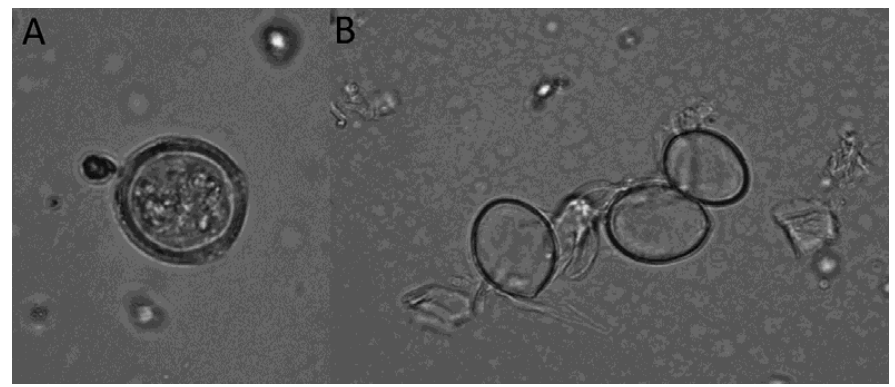

Fig. 5. A, Oospore and B, sporangia, likely of Peronospora variabilis, from quinoa seed wash.

TABLE 3. Primers used in this study

\begin{tabular}{|c|c|c|}
\hline Primer name & Sequence $\left(5^{\prime}\right.$ to $\left.3^{\prime}\right)$ & Source \\
\hline PV6F & GTTGCTGGTTGTGAAGGCTG & This study \\
\hline PV6R & ATGCTACGCAACCGAAGTCA & This study \\
\hline DC6 & GAGGGACTTTTGGGTAATCA & 7 \\
\hline ITS4 & TCCTCCGCTTATTGATATGC & 36 \\
\hline ITS6 & GAAGGTGAAGTCGTAACAAGG & 36 \\
\hline Peronosporomycete forward & GGCAAATGGGTTTTCAAGATCC & 22 \\
\hline Peronosporomycete reverse & CCATGATTAATACCACAAATTTCACTAC & 22 \\
\hline
\end{tabular}


mildew that was collected from Chenopodium weed species in Ecuador (from $C$. album [unfilled boxes]: ECDM19 and -68 and from Chenopodium sp. [black boxes]: ECDM16, -69, and -71), which may indicate that weedy Chenopodium spp. can be a reservoir of the pathogen. The only samples that were not included in the quinoa downy mildew $P$. variabilis clade were samples ECPV9, ECPV10, and ECColtaSystemic, collected in Ecuador from quinoa that did not show the characteristic symptoms of typical downy mildew but of a systemic-like infection. These samples were grouped in a separate clade (100\% bootstrap support) more closely related to $P$. boni-herici and $P$. farinosa f. sp. spinaciae, and are a separate species from $P$. variabilis.
For the ML tree generated by $C O X 2$ sequences (Fig. 6), there was a major clade that included all samples of quinoa downy mildew; however, there was a clear separation of the U.S. lineage from the South American samples (97\% bootstrap support). RS, a $P$. variabilis line collected in Pennsylvania, is phylogenetically placed within the South American lineage, whereas LV, the other $P$. variabilis line collected in Pennsylvania, is closest to the U.S. lineage. As per the ITS phylogeny, samples ECPV9, ECPV10, and ECColtaSystemic from Ecuador were placed in a separate clade, with $100 \%$ bootstrap support. The clear separation from the main quinoa downy mildew pathogen in both phylogenies indicates that samples ECPV9, ECPV10, and ECColtaSystemic

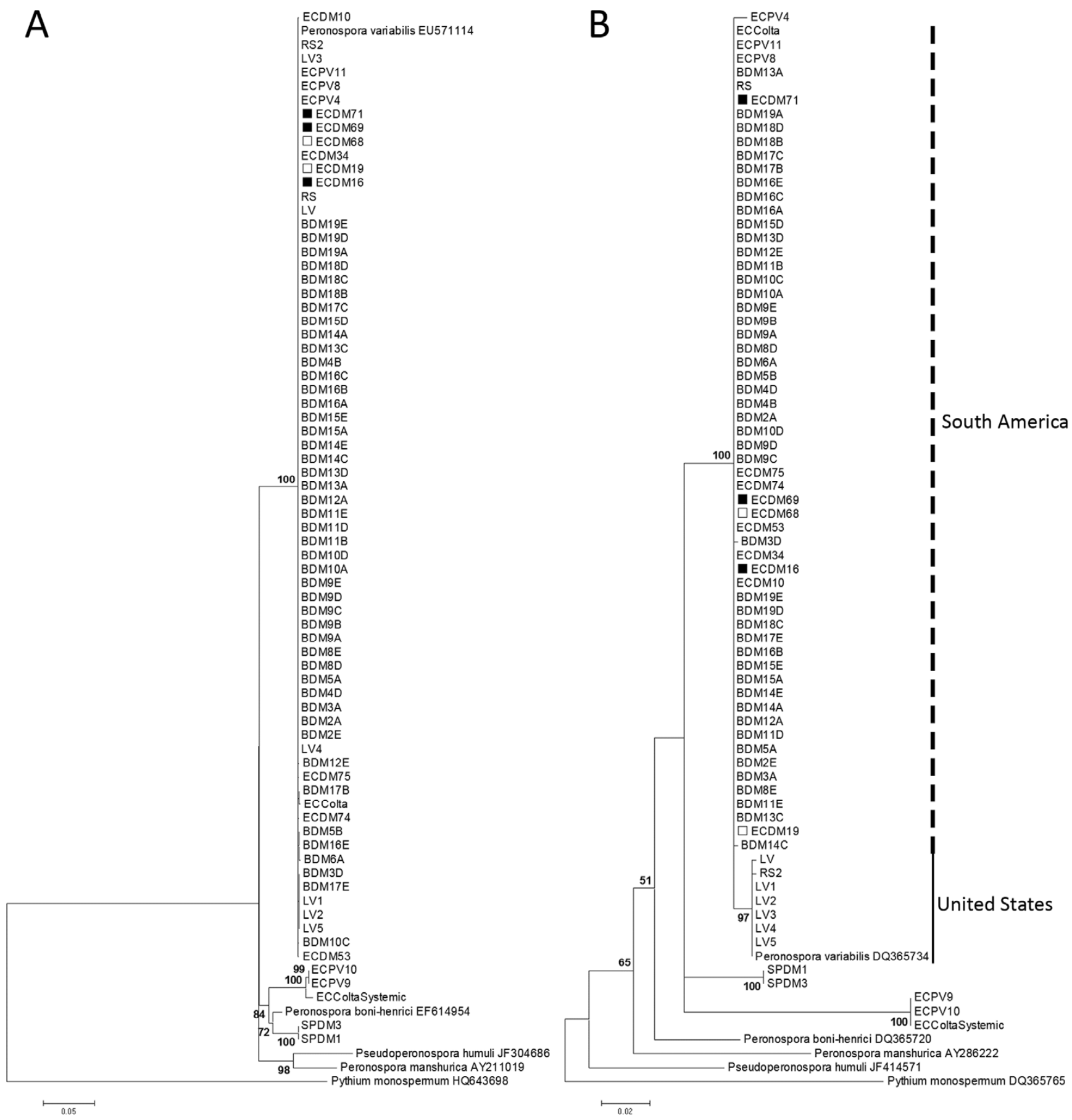

Fig. 6. A, Maximum likelihood (ML) tree of internal transcribed spacer sequences of the downy mildew pathogen, Peronospora variabilis, on Chenopodium quinoa. B, ML tree of cytochrome $c$ oxidase subunit 2 sequences of the downy mildew pathogen on C. quinoa. For both trees, sequences from either $C$. album ( $\square$ ) or Chenopodium sp. (-) collected in Ecuador are specially marked. BDM samples are from Bolivia, ECDM and ECPV are samples collected in Ecuador, while LV and RS are U.S. samples. SPDM sequences are from the spinach downy mildew pathogen, P. farinosa f. sp. spinaciae. Taxa with GenBank accession numbers were taken from other studies of Peronospora phylogeny for comparison. 
are a separate species from $P$ variabilis. For both ITS and COX2 phylogenies, no within-field or within-country differences were observed for $P$. variabilis populations.

\section{DISCUSSION}

In this research, we developed a PCR-based molecular method for the identification of $P$. variabilis, the causal agent of quinoa downy mildew. This methodology allows for the rapid, highthroughput screening of quinoa seed for the presence of $P$. variabilis. This methodology is the first step in creating certified $P$. variabilis-free quinoa seed. Use of the seed wash allows for screening of many more seed than other methodologies that require direct grinding and a DNA extraction method. The seed wash method was utilized to reduce the numerous inhibitory compounds, such as phenolic compounds in seed, which could interfere with DNA amplification. Generally, sequencing supported the findings of the PCR-based detection method, except for two seed lots (SAH and SIS10), where sequencing repeatedly amplified nontarget fungal DNA, which may have hindered detection of $P$. variabilis. Only one seed lot (NQGO) tested negative in the PCR-based assay but positive in the sequencingbased assay (Table 1), whereas seed from $C$. pallidicaule tested positive once in the PCR-based assay but $P$. variabilis was never detected in the sequencing-based method.

$P$. variabilis was detected in nearly every seed lot of quinoa that was screened. All but two quinoa seed lots (SIS10 and SIS11) were sold for direct human consumption, which means the seed underwent extensive post-harvest processing to remove saponins in the quinoa seed coat (15). Saponins are removed either by washing or abrasion (15), and this intensive processing has been shown to reduce fungal contamination of seed (31). However, the effects of the desaponification process on P. variabilis contamination are unknown. Danielsen et al. (14) visually detected oospores in the pericarp of quinoa seed in $15 \%$ of lots observed. We detected P. variabilis in $94 \%$ of seed lots tested. The seed wash procedure screens 1,300 to 1,500 seeds simultaneously versus visually screening 100 seed coats at a time. However, our test does not indicate the viability or cell type of $P$. variabilis that is detected. Viability of $P$. variabilis needs to be determined by growing seedlings under disease-conducive conditions, and these assays are time consuming. In some seed washes, we visually observed oospores and, in one seed lot, we also observed sporangia (Fig. 5). Visual inspection of seed washes was time consuming and oospores were difficult to detect due to the similarity in size, shape, and color to Chenopodium pollen and other seed debris (data not shown).

Based on ITS and COX2 phylogenies of this study's quinoa downy mildew samples, we can infer more about the pathogen population in South America and the United States. First, based on the COX2 phylogeny, there is support for geographic diversity in the pathogen. Samples collected in the United States form a clade (with strong bootstrap support, 97\%) distinct from South American samples. U.S. and South American samples form a separate clade from samples with systemic-like downy mildew symptoms (Fig. 1).

Based on ITS and COX2 phylogenies, Peronospora spp. infecting weedy Chenopodium spp. in Ecuador appear to be genetically identical to $P$. variabilis that infects quinoa. Chenopodium weeds may serve as reservoirs of $P$. variabilis between quinoa production seasons. In Pennsylvania, there was never any indication of $P$. variabilis infections of $C$. album under field conditions. Genetic markers with finer resolution than ITS and COX2 are needed to detect differences in virulence groups (30) and mating types of $P$. variabilis (9), and there may still be differences in $P$. variabilis populations from quinoa and weedy Chenopodium spp. These genetic markers could help quinoa breeders rapidly characterize local $P$. variabilis populations.
This study was initiated to determine how quinoa downy mildew was introduced to Pennsylvania in 2011 (33). It is likely that $P$. variabilis was introduced into Pennsylvania on infected quinoa seed. There are several lines of evidence to support this. First, downy mildew-like symptoms were never observed on lambsquarters adjacent to heavily sporulating quinoa plants. Other authors have noted downy mildew-like symptoms on Chenopodium weeds growing next to infected quinoa $(12,34)$. $C$. album, grown from locally collected seed, never developed symptoms of downy mildew during pathogenicity assays under controlled conditions (data not shown). It appears that there were at least two separate introductions of the pathogen to Pennsylvania. $P$. variabilis line $\mathrm{LV}$ is genetically similar to the U.S. lineage determined by the COX2 phylogeny, whereas $P$. variabilis line RS was more genetically similar to the South American COX2 lineage. It should be noted that another sample (RS2) of $P$. variabilis collected at Rock Springs was most similar to the U.S. COX2 lineage. Oospores of $P$. variabilis were observed in leaves collected at Rock Springs which, because $P$. variabilis is heterothallic (9), supports that two separate introductions of the pathogen occurred at this single Pennsylvania field site. Further, the LV and RS lines formed oospores in mating experiments (14) and represent two mating types from two geographically distinct sites in Pennsylvania. Therefore, there were likely at least two introductions of $P$. variabilis to Pennsylvania, one from seed from South America and one from $P$. variabilis of the North American lineage, possibly from seed from North America.

Genetically isolated populations of quinoa may harbor genetically distinct populations of the causal agent of quinoa downy mildew. Quinoa plants with unique symptoms were observed in Colta Canton, Chimborazo Province, Ecuador, a region of indigenous quinoa cultivation isolated from other quinoaproducing regions, in 2011 (ECPV9 and ECPV10) and 2013 (ECColtaSystemic). These plants exhibited stunting, leaf cupping and distortion, foliar chlorosis, and extremely dense sporulation on the undersides of leaves. The symptoms on these plants were distinct from typical symptoms of quinoa downy mildew (Fig. 1) and conidiophores collected from these plants are morphologically different from $P$. variabilis (Fig. 1). These atypical symptoms were thought to be due to systemic infections originating from oospores that infected seed or seedlings $(1,18)$ but our findings suggest these samples were infected with a Peronospora $\mathrm{sp}$. that is genetically distinct from $P$. variabilis. It should be noted that quinoa leaf samples exhibiting typical downy mildew samples collected in the same fields in 2011 (ECPV4, -8, and -11) and 2013 (ECColta) were genetically similar to other South American P. variabilis samples. As is indicated by our phylogenetic analyses, the samples collected in Colta Canton may represent an undescribed species of Peronospora that also causes a downy mildew on quinoa. Comparisons of ITS sequences from this undescribed Peronospora sp. to known Peronospora spp. in GenBank have only $96 \%$ maximum identity ( $P$. pulveracea FJ384778 and $P$. corydalis AF528562) and 94 to $95 \%$ maximum identity with $P$. farinosa from $C$. album (AF528556 and AF528557). The ecology of this species of Peronospora is unknown at this time but deserves further research to determine its role in quinoa downy mildew epidemics, especially because this Peronospora sp. was demonstrated to occur in the same field as $P$. variabilis.

Demand for quinoa will not decrease and sustainable disease management strategies must be developed to ensure continued quinoa production in Andean countries. The methodology developed in this research will allow for rapid screening of quinoa seed to detect $P$. variabilis. Exclusion of pathogens from production fields is a key step in integrated management of any disease, and this research allows for the development of $P$. variabilisfree quinoa seed lots. Our findings also support previous research that indicated geographic differences in $P$. variabilis populations 
from quinoa $(5,13)$. Further, a new species of Peronospora was found in Ecuador that causes a systemic-like downy mildew infection in quinoa.

This study highlights the importance and ubiquity of seedborne $P$. variabilis in quinoa. Seedborne $P$. variabilis likely plays important roles in the spread of $P$. variabilis to new quinoaproducing regions of the world and the maintenance of $P$. variabilis in endemic regions.

\section{ACKNOWLEDGMENTS}

This research was funded by the United States Agency for International Development IPM CRSP (EPP-A-00-04-00016-00) and SANREM CRSP (EPP-A-00-00-0013-00). We thank G. Plata, PROINPA Foundation, for preparation of Bolivian quinoa samples, and B. Gugino for a critical review of the manuscript.

\section{LITERATURE CITED}

1. Alandia, S., Otazu, V., and Salas, B. 1979. Enfermedades. Pages 137-148 in: La quinua y la kañiwa: cultivos andinos. M. Tapia, ed. Bib. Orton IICA/CATIE, Bogata, Columbia.

2. Azevedo, M. I., Botton, S. A., Pereira, D. I. B., Robe, L. J., Jesus, F. P. K., Mahl, C. D., Costa, M. M., Alves, S. H., and Santurio, J. M. 2012. Phylogenetic relationships of Brazilian isolates of Pythium insidiosum based on ITS rDNA and cytochrome oxidase II gene sequences. Vet. Microbiol. 159:141-148.

3. Belbahri, L., Calmin, G., Pawlowski, J., and Lefort, F. 2005. Phylogenetic analysis and real time PCR detection of a presumably undescribed Peronospora species on sweet basil and sage. Mycol. Res. 109:1276-1287.

4. Bhargava, A. 2006. Chenopodium quinoa-an Indian perspective. Ind. Crops Prod. 23:73-87.

5. Choi, Y. J., Danielsen, S., Lubeck, M., Hong, S. B., Delhey, R., and Shin, H. D. 2010. Morphological and molecular characterization of the causal agent of downy mildew on quinoa (Chenopodium quinoa). Mycopathologia 169:403-412.

6. Choi, Y. J., Denchev, C. M., and Shin, H. D. 2008. Morphological and molecular analyses support existence of host-specific Peronospora species infecting Chenopodium. Mycopathologia 165:155-164.

7. Cooke, D. E. L., Drenth, A., Duncan, J. M., Wagels, G., and Brasier, C. M. 2000. A molecular phylogeny of Phytophthora and related oomycetes. Fungal Genet. Biol. 30:17-32.

8. Cusack, D. F. 1984. Quinua: grain of the Incas. Ecologist 14:21-31.

9. Danielsen, S. 2001. Heterothallism in Peronospora farinosa f. sp. chenopodii, the causal agent of downy mildew of quinoa (Chenopodium quinoa). J. Basic Microbiol. 41:305-308.

10. Danielsen, S., Bonifacio, A., and Ames, T. 2003. Diseases of quinoa (Chenopodium quinoa). Food Rev. Int. 19:43-59.

11. Danielsen, S., Jacobsen, S. E., Echegaray, J., and Ames, T. 1999. Impact of downy mildew on the yield of quinoa. CIP Program Rep. 19992000:397-401.

12. Danielsen, S., Jacobsen, S. E., and Hockenhull, J. 2002. First report of downy mildew of quinoa caused by Peronospora farinosa f. sp. chenopodii in Denmark. Plant Dis. 86:1175.

13. Danielsen, S., and Lubeck, M. 2010. Universally primed-PCR indicates geographical variation of Peronospora farinosa ex. Chenopodium quinoa. J. Basic Microbiol. 50:104-109.

14. Danielsen, S., Mercado, V. H., Ames, T., and Munk, L. 2004. Seed transmission of downy mildew (Peronospora farinosa f. sp. chenopodii) in quinoa and effect of relative humidity on seedling infection. Seed Sci. Technol. 32:91-98.

15. Dominguez, S. S. 2003. Quinoa-postharvest and commercialization. Food Rev. Int. 19:191-201.

16. Edgar, R. C. 2004. MUSCLE: multiple sequence alignment with high accuracy and high throughput. Nucleic Acids Res. 32:1792-1797.
17. El Hafid, R., Aitelmaalem, H., Driedger, D., Bandara, M., and Stevenson, J. 2005. Quinoa...The Next Cinderella Crop for Alberta? Alberta Agriculture, Food and Rural Development Ag Entrepreneurship, Edmonton, Alberta, Canada.

18. Frinking, H. D., and Linders, E. G. A. 1986. A comparison of two pathosystems: Downy mildew on Spinacia oleracea and on Chenopodium album. Neth. J. Plant Pathol. 92:97-106.

19. Göker, M., Garcia-Blazquez, G., Voglmayr, H., Telleria, M. T., and Martin, M. P. 2009. Molecular taxonomy of phytopathogenic fungi: A case study in Peronospora. PLoS One 4:e6319.

20. Göker, M., Voglmayra, H., Riethmuller, A., and Oberwinkler, F. 2007. How do obligate parasites evolve? A multi-gene phylogenetic analysis of downy mildews. Fungal Genet. Biol. 44:105-122.

21. Göker, M., Voglmayr, H., Riethmuller, A., Weiss, M., and Oberwinkler, F. 2003. Taxonomic aspects of Peronosporaceae inferred from Bayesian molecular phylogenetics. Can. J. Bot. 81:672-683.

22. Hudspeth, D. S. S., Nadler, S. A., and Hudspeth, M. E. S. 2000. A COX2 molecular phylogeny of the Peronosporomycetes. Mycologia 92:674-684.

23. Hudspeth, D. S. S., Stenger, D., and Hudspeth, M. E. S. 2003. A cox2 phylogenetic hypothesis for the downy mildews and white rusts. Fungal Divers. 13:47-57.

24. Inaba, T., Takahashi, K., and Morinaka, T. 1983. Seed transmission of spinach downy mildew. Plant Dis. 67:1139-1141.

25. Jacobsen, S. E. 1998. Developmental stability of quinoa under European conditions. Ind. Crops Prod. 7:169-174.

26. Korbie, D. J., and Mattick, J. S. 2008. Touchdown PCR for increased specificity and sensitivity in PCR amplification. Nat. Protocols 3:14521456.

27. Kroon, L. P. N. M., Bakker, F. T., van den Bosch, G. B. M., Bonants, P. J. M., and Flier, W. G. 2004. Phylogenetic analysis of Phytophthora species based on mitochondial and nuclear DNA sequences. Fungal Genet. Biol. 41:766-782.

28. Kumar, A., Bhargava, A., Shukla, S., Singh, H. B., and Ohri, D. 2006. Screening of exotic Chenopodium quinoa accessions for downy mildew resistance under mid-eastern conditions of India. Crop Prot. 25:879-889.

29. Landa, B. B., Montes-Borrego, M., Munoz-Lesma, F. J., and JimenezDiaz, R. M. 2007. Phylogenetic analysis of downy mildew pathogens of opium poppy and PCR-based in plant and seed detection of Peronospora arborescens. Phytopathology 97:1380-1390.

30. Ochoa, J., Frinking, H. D., and Jacobs, T. 1999. Postulation of virulence groups and resistance factors in the quinoa downy mildew pathosystem using material from Ecuador. Plant Pathol. 48:425-430.

31. Pappier, U., Fernandez Pinto, V., Larumbe, G., and Vaamonde, G. 2008. Effect of processing for saponin removal on fungal contamination of quinoa seeds (Chenopodium quinoa Willd.). Int. J. Food Microbiol. 125:153-157.

32. Tamura, K., Peterson, D., Peterson, N., Stecher, G., Nei, M., and Kumar, S. 2011. MEGA5: Molecular evolutionary genetics analysis using maximum likelihood, evolutionary distance, and maximum parsimony method. Mol. Biol. Evol. 28:2731-2739.

33. Testen, A. L., McKemy, J. M., and Backman, P. A. 2012. First report of quinoa downy mildew caused by Peronospora variabilis in the United States. Plant Dis. 96:146.

34. Tewari, J. P., and Boyetchko, S. M. 1990. Occurrence of Peronospora farinosa f. sp. chenopodii on quinoa in Canada. Can. Plant Dis. Surv. 70:127-128.

35. UNFAO. 2011. FAOSTAT. Food and Agriculture Organization of the United Nations, FAOSTAT database. http://faostat.fao.org

36. White, T. J., Bruns, T., Lee, S., and Taylor, J. 1990. Amplification and direct sequencing of fungal ribosomal RNA genes for phylogenetics. Pages 315-322 in: PCR Protocols: A Guide to Methods and Applications. M. A. Innis, D. H. Gelfand, J. J. Sninsky, and T. J. White, eds. Academic Press, San Diego, CA.

37. Ye, J., Coulouris, G., Zaretskaya, I., Cutcutache, I., Rozen, S., and Madden, T. 2012. Primer-BLAST: A tool to design target-specific primers for polymerase chain reaction. BMC Bioinf. 13:134. 